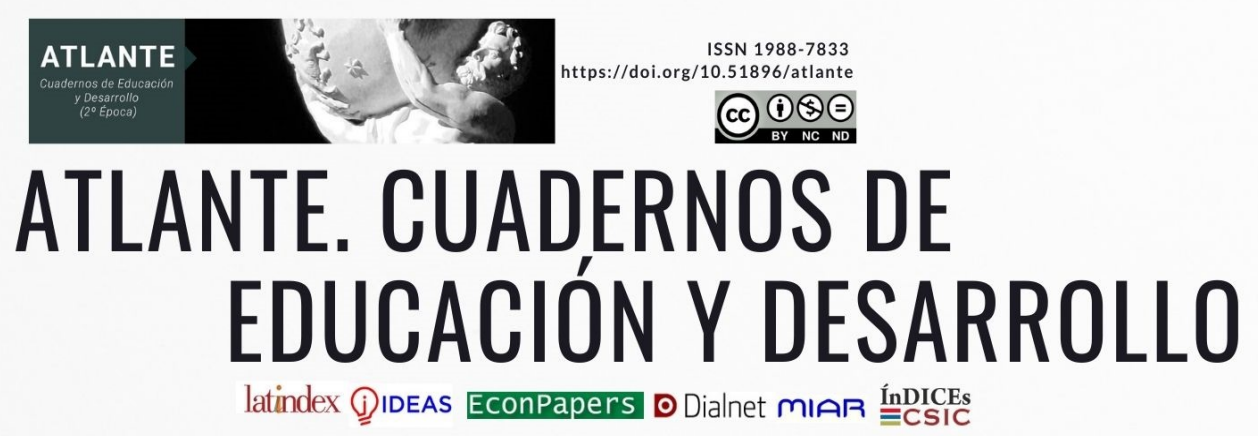

\title{
LAS ADAPTACIONES CURRICULARES Y SU APLICABILIDAD PARA LA EDUCACIÓN INCLUSIVA EN EL CANTÓN SANTA ANA 2020
}

\author{
Melissa Rosario Molina Vinces' \\ Estudiante egresada de la escuela de Trabajo Social de la Universidad Técnica de Manabí-Ecuador \\ Correo: melissamolina_125@hotmail.com \\ Mercedes Cedeño Barreto, MG. ${ }^{2}$ \\ Tutora y docente principal de la carrera de Trabajo social de la Universidad Técnica de Manabí-Ecuador \\ Correo: mercedes.cedeno@utm.edu.ec 0990193424
}

Para citar este artículo puede utilizar el siguiente formato:

Melissa Rosario Molina Vinces y Mercedes Cedeño Barreto: "Las adaptaciones curriculares y su aplicabilidad para la educación inclusiva en el Cantón Santa Ana 2020", Revista Atlante: Cuadernos de Educación y Desarrollo (vol 13, № 2 febrero 2021, pp. 93-112). En línea:

https://www.eumed.net/es/revistas/atlante/2021-febrero/adaptaciones-curriculares-educacion

\section{Resumen}

La educación ha tenido cambios significativos a nivel mundial, que han permitido disminuir la discriminación y exclusión de aquellos estudiantes que presentaban problemas de aprendizaje o algún tipo de discapacidad, en Ecuador se han creado leyes, normativas y se han aplicado estrategias como las adaptaciones curriculares un instrumento fundamental en la educación inclusiva, es por ello que el objetivo de esta investigación es analizar las adaptaciones curriculares y su aplicabilidad para la educación inclusiva en el cantón Santa Ana 2020, misma que se realizó aplicando una metodología de corte descriptivo con enfoque cuantitativo, la utilización de métodos y técnicas que contribuyeron a la obtención de información y el análisis de los datos obtenidos. Los resultados evidenciaron que las instituciones investigadas cumplen con todo el proceso de detección, valoración y aplicación de las adaptaciones curriculares a estudiantes con N.E.E o discapacidad según lo establece el Ministerio de Educación, contando con el apoyo de instituciones estatales y comunidad educativa existiendo un buen clima institucional de acuerdo a los profesionales del DECE entrevistados. En la encuesta aplicada a los docentes consideran que su preparación es buena capacitándose permanentemente para atender estos casos, algunos expresaron que no existe la apropiada infraestructura de accesibilidad y el mobiliario adecuado en las aulas que permitan la movilidad a los estudiantes con discapacidad. El rendimiento de 
los estudiantes es muy bueno al aplicar una metodología acorde a sus capacidades y necesidades ya que no todos aprenden al mismo ritmo que los niños regulares.

Palabras claves: adaptaciones curriculares, educación inclusiva, estudiantes, necesidades educativas especiales, discapacidad.

\title{
CURRICULUM ADAPTATIONS AND THEIR APPLICABILITY FOR INCLUSIVE EDUCATION IN THE CANTON OF SANTA ANA 2020
}

\begin{abstract}
Education has undergone significant changes worldwide, which have allowed to diminish the discrimination and exclusion of those students with learning problems or some type of disability, in Ecuador laws have been created. regulations and strategies such as curricular adaptations have been applied as a fundamental instrument in inclusive education, this is why the objective of this research is to analyze the curricular adaptations and their applicability for inclusive education in the canton of Santa Ana 2020, which was carried out using a descriptive cutting methodology with a quantitative approach, the use of methods and techniques that contributed to the collection of information and analysis of the data obtained. The results showed that the institutions investigated comply with the whole process of detection, evaluation and application of curricular adaptations to students with N.E.E or disability as established by the Ministry of Education, With the support of state institutions and educational community there exists a good institutional climate according to the DECE professionals interviewed. In the survey applied to the teachers, they consider that their preparation is good and that they are permanently trained to attend these cases, some of them expressed that there is no appropriate accessibility infrastructure and adequate furniture in the classrooms to allow mobility to students with disabilities. Students' performance is very good at applying a methodology that matches their abilities and needs as not everyone learns at the same pace as regular children.
\end{abstract}

Keywords: curricular adaptations, inclusive education, students, special educational needs, disability.

\section{Introducción}

El siglo XXI se enfrenta a diferentes cambios en distintos ámbitos para responder a los desafíos a situaciones o acontecimientos que pueden ser negativos dentro de la sociedad. Según la UNESCO (2015) citado por Rodríguez Hernández (2016), la educación en el mundo cumple un rol fundamental al promover los derechos humanos y la dignidad con el fin de erradicar la pobreza y construir un mejor futuro para todos. 
En muchos paises de América Latina y del contexto Europeo según Payà (2010), todavía existe una brecha entre las propuestas que se hacen mediante los discursos teóricos con las realidades prácticas de los programas que se implementan. Considera que la participación ciudadana es escencial para las politicas públicas con perspectiva de desarrollo y bienestar social, además la corresponsabilidad de la comunidad educativa que es escencial para lograr una educación inclusiva de calidad y para todos.

En Ecuador se han creado diferentes leyes y normativas las cuales se detallarán en el desarrollo de este artículo como la Constitución de la República del Ecuador en 2008, la Ley Orgánica De Educación Intercultural, el Reglamento General a la Ley Orgánica de Educación Intercultural y el Código de la Niñez y Adolescencia, que garantizan la educación como un derecho de las personas a lo largo de su vida, la igualdad e inclusión sin discriminación alguna. Así mismo el sistema educativo juega un papel importante en la aplicación de diferentes propuestas educacionales y pedagógicas dirigidos a los niños, niñas y adolescentes que poseen una discapacidad o presenten una necesidad educativa especial que les permita obtener mayores oportunidades de aprendizaje en su proceso de enseñanza. Por ello el principal objetivo de este artículo es analizar las adaptaciones curriculares y su aplicabilidad para la educación inclusiva en el cantón Santa Ana 2020, utilizando una metodología adecuada para obtener la información necesaria.

Dentro de las instituciones educativas existe el apoyo del DECE (Departamento de Consejería Estudiantil) y de la UDAI (Unidad Distrital de Apoyo a la Inclusión) que garantizan el proceso de adaptacion curricular según lo establece el Ministerio de Educación. Las adaptaciones curriculares son un instrumento importante en la educación integral por ello Paniagua (2005), expresa que "tenderán a posibilitar el acceso al currículo común, o a brindar aprendizajes equivalentes por su temática, profundidad y riqueza a los niños con necesidades educativas especiales". Este instrumento permitirá que la enseñanza sea adaptada en cuanto a los objetivos, destrezas, metodología, evaluación, condiciones de acceso entre otros elementos de acuerdo a las necesidades y capacidades de los estudiantes en la que es conveniente realizar una detección y valoración previa al estudiante para identificar qué tipo de adaptación requiere y así lograr una verdadera educación inclusiva.

Existen elementos imprescindibles para facilitar el proceso de aplicación de adaptación curricular en la educación inclusiva los cuales se detallaran en el desarrollo de este artículo como por ejemplo el mobiliario e infraestructura adecuada, docentes preparados para atender a los estudiantes que presenten problemas de aprendizaje, buen clima institucional para coordinar con la comunidad educativa y los organismos estatales tanto internos como externos el apoyo necesario en beneficio de los estudiantes.

La escuela inclusiva tiene como objetivo garantizar la permanencia de todos los estudiantes independientemente de que tengan o no alguna discapacidad logrando una mejor educación. "La Educación Inclusiva implica la creación de condiciones idóneas y realizables para alcanzar la oportunidad de aprendizaje de todos, apela a manera que se comprende y se respeta la diversidad" 
(León Guerrero, 2011). Es importante promover una sociedad más justa, con igualdad de oportunidades también la escuela inclusiva es una solución a problemas que solo la educación puede brindar en un mundo globalizado.

En la educación inclusiva se requiere el apoyo de las instituciones estatales y de toda la comunidad educativa, los docentes cumplen un rol muy importante deben de estar correctamente capacitados para atender las NEE y con el compromiso de tener una buena actitud para propiciar una enseñanza acorde a las insuficiencias que se presenten. En la medida que el profesor asuma su responsabilidad en la formación de los educandos manifestará actitudes más apropiadas ante las NEE; es decir, si el docente está preparado correctamente podrá enseñar de la manera correcta a estos alumnos. (Alejandro Conteno, Erraéz Alvardo, Vargas Gaona, \& Espinoza Freire, 2018)

\section{Desarrollo}

\section{Adaptaciones curriculares}

La educación se enfrenta a un gran desafío en la actualidad y tiene que ver con descubrir los modos de enseñanza y estrategias que permitan responder a las necesidades y diferencias individuales de los estudiantes que aseguren el éxito del aprendizaje y su derecho a recibir una educación de calidad.

El Ministerio de Educación en Ecuador tiene el difícil reto de alcanzar una calidad educativa, evitar la discriminación, desigualdad de oportunidades, respetar las características y las necesidades individuales de todos los estudiantes con o sin discapacidad, además implica la transformación en las prácticas metodológicas que conducirán a la transformación de la educación. Por ello se han implementado diversas normas y leyes para favorecer a aquellos grupos prioritarios en la educación, por ejemplo el Código de la Niñez y Adolescencia (2017), que en su artículo 37 inciso 3 tipifica que el sistema educativo debe contemplar propuestas educacionales flexibles y alternativas para atender las necesidades de todos los niños, niñas y adolescentes, con prioridad a los que poseen discapacidad, trabajan o viven una situación que requiera mayores oportunidades para aprender.

Para lograr una educación de calidad se debe de contar con la colaboración de toda la comunidad educativa conformada por actores vinculados directamente a la institución como padres de familia, docentes, autoridades, personal de servicio y administrativo, estudiantes, que son pieza clave en la educación inclusiva, por ello Posada Silva, W.Y.; Marín Giraldo, Y. y Gómez Chica, S (2015), expresa que los directivos y docentes de las instituciones deben de tener conocimientos sobre la aplicación de las adaptaciones para que puedan ser implementadas.

Además de lo antes mencionado, dentro del proceso inclusivo es fundamental contar con el apoyo de las Unidades Distritales de Apoyo a la Inclusión (UDAI), creadas para atender las NEE asociadas o no a una discapacidad, ubicadas en cada Distrito Educativo a nivel nacional y se encargan 
de la evaluación, asesoramiento e intervención psicopedagógica en todas las modalidades de atención y niveles del sistema educativo (Ministerio de Educación del Ecuador, 2020), la función de las unidades de apoyo es brindar asesoría a todos los docentes en el proceso de las adaptaciones curriculares, priorizando las destrezas básicas de cada estudiante. .

Referente a la definición de las adaptaciones curriculares que son la base de una educación integral dentro de las instituciones, Galve y Trallero (2002), consideran que son un instrumento fundamental para la enseñanza, además son modificaciones que se realizan al curriculum escolar, objetivos, destrezas, metodología, evaluación, condiciones de acceso entre otros elementos para que la enseñanza sea adaptada a las necesidades y diferencias del alumnado.

Existen diferentes tipos de adaptaciones según lo expresa el Ministerio de Educación del Ecuador (2016), las cuales son:

\section{$\checkmark$ Según nivel de concreción:}

- Primer nivel (macrocurrículo): Es el modelo curricular elaborado por un estado o gobierno.

- Segundo nivel (mesocurrículo): Hace referencia a lo que la institución educativa planifica. En este caso al Proyecto Educativo Institucional, que contiene la Planificación Curricular Institucional, a la cual se articula la Planificación Curricular Anual.

- Tercer nivel (microcurrículo): Este tipo de adaptaciones se desprende del mesocurrículo y se ajusta a las necesidades y particularidades de los estudiantes.

$\checkmark$ Según el ente en que se aplica:

- Centro educativo: Ajustar el currículo nacional al de la institución a su contexto y necesidades.

- Aula: Estudiantes de cualquier grado o curso de EGB o BGU, según se requiera.

- Individuo: Cuando la adaptación se la realiza para un estudiante en particular.

$\checkmark$ Según grado de afectación:

- Grado 1 o de acceso: Modificaciones en infraestructura, recursos materiales y humanos, de comunicación y de tiempo.

- Grado 2 o no significativa: Metodología y evaluación deben ser flexibles y adaptables a cada estudiante.

- Grado 3 o significativa: Modificaciones en los objetivos educativos y destrezas con criterios de desempeño, de acuerdo a la capacidad del estudiante.

$\checkmark$ Según duración:

- Temporales: Establecidas en un tiempo determinado, aplicado a estudiantes con NEE no asociadas a la discapacidad.

- Permanentes: Permanecen durante todo el proceso escolar del estudiante, aplicadas a estudiantes con NEE asociadas a la discapacidad. 
Es esencial identificar el tipo de necesidad que presenta el alumno, antes de aplicar una adaptación curricular por lo que es necesario definir el concepto de Necesidades Educativas Especiales, ante ello es preciso mencionar el Reglamento General a la Ley Orgánica de Educación Intercultural (2012) que en el artículo 228 establece que:

Son estudiantes con necesidades educativas especiales aquellos que requieren apoyo 0 adaptaciones temporales o permanentes que les permitan o acceder a un servicio de calidad de acuerdo a su condición. Estos apoyos y adaptaciones pueden ser de aprendizaje, de accesibilidad o de comunicación.

Dentro del Reglamento y artículo antes mencionado, también se estipula que existen dos tipos de necesidades educativas especiales:

Necesidades educativas especiales no asociadas a la discapacidad:

1. Dificultades específicas de aprendizaje: dislexia, discalculia, disgrafía, disortografía, disfasia, trastornos por déficit de atención e hiperactividad, trastornos del comportamiento, entre otras dificultades.

2. Situaciones de vulnerabilidad: enfermedades catastróficas, movilidad humana, menores infractores, víctimas de violencia, adicciones y otras situaciones excepcionales previstas en el presente reglamento.

3. Dotación superior: altas capacidades intelectuales.

Necesidades educativas especiales asociadas a la discapacidad:

1. Discapacidad intelectual, física-motriz, auditiva, visual o mental;

2. Multidiscapacidades; y,

3. Trastornos generalizados del desarrollo (Autismo, síndrome de Asperger, síndrome de Rett, entre otros).

Antes de empezar con el proceso que se debe realizar para la aplicación de las adaptaciones curriculares es conveniente identificar si la institución cuenta con el entorno de aprendizaje adecuado para garantizar el proceso de inclusión, esta evaluación la llevan a cabo las autoridades del plantel y docentes, en efecto el Ministerio de Educación del Ecuador propone valorar los siguientes aspectos:

- Accesibilidad física. Evaluar si los espacios son flexibles, abiertos y fáciles de transitar.

- Proyecto curricular. Se establece si en la Planificación Curricular Institucional (PCl) toman en cuenta a los estudiantes con NEE y como aplican las adaptaciones curriculares.

- Recursos materiales. Valorar el tipo y la variedad de recursos que posee la institución para estudiantes con NEE.

- Recursos personales. Determinar con que personal cuenta dentro de la institución. (DECE, docentes, autoridades). 
- Formación del profesorado en áreas afines. Los docentes deben de tener estudios en pedagogía, psicología, o cursos de actualización en referente a la Educación Inclusiva.

- Actitud de padres, docentes y estudiantes. Evaluar la colaboración de los padres de familia en todo el proceso educativo, la disposición del docente y las actitudes de los compañeros a un estudiante con NEE.

Asimismo, se debe evaluar el aula:

- Aspectos físicos y de organización del espacio. Organización del espacio y distribución del tiempo, y las condiciones del aula: iluminación, mobiliario, amplitud, entre otras.

- Recursos materiales y personales. Se identifica si la institución cuenta con el material didáctico requerido para el estudiante con NEE, y si cuenta con un DECE.

- Programación. Se considera realizar en caso de ser necesario una planificación individualizada para el estudiante con NEE, elaboración de documentos como el DIAC, la Planificación de Aula y el Plan de Acompañamiento.

- Método de aprendizaje. Revisión de la metodología utilizada, los aprendizajes previos que posea el estudiante son de vital importancia.

- Actividades de enseñanza-aprendizaje. Evaluar la calidad y cantidad de actividades como trabajos individuales, grupales, que fomentan el aprendizaje del estudiante.

- Relaciones. Se evalúa el clima institucional es decir, las relaciones entre los miembros de la comunidad educativa.

Dentro de la Guia de trabajo sobre las adaptaciones curriculares para la educación inclusiva del Ministerio de Educación del Ecuador (2016), habla sobre el proceso que se aplica para realizar las adaptaciones curriculares al estudiante que presenta problemas de aprendizaje que posiblemente manifieste una NEE, por ellos se debe iniciar con la detección y valoración tomando en cuenta el contexto familiar, escolar y social. EI DECE solicita al docente llenar un cuestionario sobre las principales dificultades de aprendizaje detectadas, denominado CEPA (Cuestionario de Evaluacion de Problemas de Aprendizaje), a su vez realizar un informe en el que recoja todas las dificultades observadas en el aula de clases sin etiquetarse al estudiante.

Estos documentos se entregan al DECE o en caso de no existir dentro de la institución fiscal se remite a la UDAI o instituciones anexas que si posean DECE. En las instituciones particulares los padres tienen la libertad de elegir un centro psicopedagógico o neuropsicológico que pueda realizar la evaluación. En las dos situaciones se debe de dar un informe completo que permita realizar las acciones pertinentes para poder determinar la necesidad de realizar adaptaciones curriculares por parte del DECE o UDAI.

Es imprescindible remitir el caso a profesionales externos para las evaluaciones e intervenciones pertinentes como lo establece el Reglamento General a la Ley Orgánica de Educación Intercultural 
(2012), en el art 61 estipula que se debe articular una red interinstitucional con otros organismos de protección como el Consejo Nacional de la Niñez y la Adolescencia, los Consejos Cantonales de Niñez y Adolescencia, la Defensoría del Pueblo, los Ministerios de Salud, de Inclusión, entre otros.

Luego de realizar la detección o valoración se convoca a los docentes titulares o jefes de área para la elaboracion del DIAC con el equipo convocado. EI DIAC es el documento individual de adapatacion curricular, instrumento de registro de los datos generales y específicos del estudiante y su contexto familiar, condiciones fisicas y afectivas que rodean al estudiante, así como las pautas de crianza (normas, hábitos y manera particular de educar a los hijos), sus necesidades educativas especiales, su competencia curricular, propuestas curricular adaptada, entre otros, para elaborar las adaptaciones curriculares necesarias. Los principales responsables de la elaboración de este documento es el docente titular con el apoyo del DECE o UDAI, mismo que debe ser archivado en el expediente del estudiante que serviran de base para las futuras adaptaciones durante la vida escolar, desde Educación Inicial hasta el Bachillerato Obligatorio. La autoridad correspondiente aprueba el DIAC y se realiza una reunion con los padres de familia para entregar este documento, ellos deben firmar una carta de aceptación de las adaptaciones curriculares efectuadas.

Un ejemplo de adaptación curricular para un estudiante con discapacidad intelectual en este caso con síndrome de Dawn que presenta una discapacidad moderada se recomienda apoyar al estudiante buscando actividades y entornos adecuados en los que interactúe socialmente y utilizar un lenguaje sencillo con palabras cortas enfatizando la entonación. Referente al acceso al currículo se deben de usar letreros con pictogramas para que el estudiante pueda ubicarse, como baño, bar, dirección, entre otros. Además utilizar materiales concretos y los Sistemas Alternativos y Aumentativos de Comunicación. En la parte metodológica en el área de matemáticas pasar de lo concreto a lo semiconcreto, en lenguaje para el vocabulario partir de lo concreto y posterior utilizar láminas, también se podría utilizar el método fonético conjugado con diferentes símbolos que pueden ser corporales o visuales. En la evaluación al estudiante con discapacidad intelectual moderada es recomendable realizarlas de manera diferenciada con pruebas objetivas y orales con instrucciones segmentadas cortas y claras. Los objetivos y destrezas con criterio de desempeño hacia el estudiante se deben de modificar de acuerdo a la competencia curricular.

El Departamento de Consejería Estudiantil conformado por Psicólogos y Trabajador (a) Social de acuerdo al número de alumnos que tiene la unidad educativa, debe de realizar un seguimiento al estudiante y de esta manera cumplir con su principal función dentro de la institución, como lo estipula el Reglamento General a la Ley Orgánica de Educación Intercultural (2012), en el articulo 60 que manifiesta que deben participar según su área profesional, en reuniones de equipos de trabajo y otros colectivos internos o externos a la institución, que permitan mantenerlos actualizados para abordar de manera correcta y efectiva los casos que se presenten como situaciones individuales, grupales e institucionales que requieran de su intervención y la UDAI realiza el respectivo control a los DECE para verificar el 
seguimiento de los casos. Para culminar este proceso se realiza una revisión de los resultados obtenidos del primer quimestre a los estudiantes para reajustar las adaptaciones en caso que lo necesite, por último se planifica una reunión con el equipo multidisciplinario para dar los resultados finales.

También se deben de tomar en consideración los siguientes instrumentos que facilitarán la adaptación curricular. La elaboración del Plan del Aula le corresponde realizarla al docente con el debido asesoramiento del DECE o UDAI en la cual se deben incluir diferentes elementos como los objetivos, contenidos, la metodología, recursos y evaluación, el procedimiento en la elaboración de este documento el docente debe referirse al modelo oficial de Planificación de Aula que contiene los datos informativos, planificación, adaptaciones curriculares que debe ser elaborado a partir del DIAC y por último la revisión y aprobación por parte de la autoridad respectiva. El Plan de Acompañamiento sintetiza toda la información del DIAC y la Planificación del Aula, mismo que orienta al docente sobre las adaptaciones del currículo que debe aplicar una vez realizado el proceso de detección de la necesidad educativa del estudiante y las adaptaciones curriculares determinadas, todos estos documentos deben de registrarse en el expediente respectivo del estudiante.

Este proceso se lo aplica en la modalidad presencial, sin embargo en el año lectivo 2020-2021 debido a la pandemia mundial provocada por el Covid-19 las escuelas cerraron y se optó por la educación online, de acuerdo con los profesionales del DECE entrevistados las adaptaciones curriculares se realizaron con el apoyo de los docentes mediante la tecnología dando el seguimiento al estudiante de manera virtual o incluso realizando visitas domiciliarias, teniendo en cuenta las evaluaciones y los informes del periodo lectivo pasado para poder continuar aplicando las adaptaciones en este nuevo periodo escolar. Los padres debido a la emergencia sanitaria se convirtieron en una pieza clave dentro de la educación de sus hijos, contribuyendo e involucrándose en la enseñanza e incluso logrando una evolución significativa en el aprendizaje de los niños, las tareas que se envían a los niños con necesidades educativas asociadas o no a una discapacidad están diseñadas de acuerdo a sus capacidades y habilidades, por ello el Ministerio de Educación del Ecuador (2020), publicó la Guía de apoyo pedagógico para la comunidad educativa durante el período de suspensión de las actividades escolares presenciales por la emergencia sanitaria covid-19 los cuales dan pautas para continuar con la educación inclusiva asegurando el derecho de los niños.

\section{Educación Inclusiva}

La educación inclusiva debe de responder ante las necesidades de todos los estudiantes independientemente si tienen discapacidad o no, desventajas y dificultades, esto permitirá romper todo tipo de barreras en el aprendizaje, realizando modificaciones en los contenidos para obtener una educación de calidad. Es por ello que Lledó Carreres \& Arnaiz Sánchez (2010), considera que la escuela del siglo XXI a nivel mundial tiene el gran reto de ser eminentemente inclusiva desde la perspectiva de atender la diversidad en las aulas. La UNESCO (2004) menciona que "concebir la educación como algo 
fundamental para el desarrollo, tanto del individuo como de la sociedad, es central en el compromiso de la educación inclusiva" (p.16).

La Declaración de Salamanca UNESCO (1994) sobre los Principios, Políticas y Prácticas para las Necesidades Educativas Especiales señala el cambio de rumbo en las escuelas promoviendo una educación inclusiva para hacer frente a las diferentes actitudes discriminatorias y de esta manera alcanzar la educación para todos (Payà 2010). Convirtiéndose en una lucha constante tratando de reducir la exclusión y discriminación en los sistemas educativos.

La normativa ecuatoriana es muy amplia en relación a garantizar el derechos a la educación de los niños, niñas y adolescentes, los cuales se mencionarán a continuación. La Constitución de la Republica del Ecuador, (2008) establece el artículo 26 establece que:

La educación es un derecho de las personas a lo largo de su vida y un deber ineludible e inexcusable del Estado. Constituye un área prioritaria de la política pública y de la inversión estatal, garantía de la igualdad e inclusión social y condición indispensable para el buen vivir; el Estado garantizará el acceso universal, permanencia, movilidad y egreso sin discriminación alguna.

La Ley Orgánica de Educación Intercultural (2011) estipula en el artículo 47 referente a la Educación para las personas con discapacidad que: "Tanto la educación formal como la no formal tomará en cuenta las necesidades educativas especiales de las personas en lo afectivo, cognitivo y psicomotriz..."

De igual manera en el artículo 48 relacionado con la educación para niñas, niños, adolescentes, y adultos con dotación superior expresa que:

Tendrán derecho a la educación especial correspondiente a sus capacidades. Se deben incluir, a las niñas, niños, adolescentes y jóvenes en las instituciones educativas del Sistema Nacional de Educación, en sus diferentes niveles y modalidades, garantizando la articulación curricular, infraestructura y materiales acordes con su dotación superior y su pertinencia cultural y lingüística.

La inclusión o Educación Inclusiva es un término que surge en los años 90 según Barrio de la Puente (2009), este pretende sustituir a la terminología integración, que predominaba en la práctica educativa. Es imprescindible velar por los derechos a la educación de personas con discapacidad que según Crosso (2014), es lo que diferencia la inclusión de la integración, si no existe inclusión puede llevar al aislamiento y generar obstáculos en la atención de las necesidades educacionales de todos los/as estudiantes. Las acciones se basan en la eliminación de barreras físicas, personales e institucionales que podrían limitar las oportunidades de aprendizaje de todos los estudiantes basándose en principios de igualdad, comprensibilidad y globalización. 
Según Calderón (2014), el concepto de inclusión reemplazó al término integración pues este permite abarcar algunos aspectos como la inserción en todos los espacios y ambientes en que el niño se vaya desarrollando, a su vez dentro del ámbito educativo se refiere a la reestructuración que se deben de realizar en las instituciones educativas para cubrir las demandas y requerimientos que necesite un niño con N.E.E como a los regulares. Existen otros ámbitos donde la palabra inclusión es muy común, Plancarte (2017) señala que a partir de la globalización todo debe estar "incluido", es por ello que esta palabra es común en ambientes como la salud, legislativo, relaciones internacionales entre otros.

Por lo tanto, la inclusión es un medio para lograr la igualdad social y una sociedad con igualdad de oportunidades, y también es una solución a una amplia gama de problemas que solo la educación puede brindar en un mundo globalizado. Llerena Companioni \& Salinas Martínez, (2018), habla sobre la igualdad de oportunidades dentro del proceso educativo y considera que es escencial en la educación de calidad, garantizando la accesibilidad física curricular, económica, a su vez implica un trato diferenciado mas no discriminatorio o excluyente.

La inclusión se basa en la identificación y eliminación de las barreras de aprendizaje, por lo que significa recopilar, cotejar y evaluar información para planificar las mejoras de políticas y prácticas en la educación. En ese mismo contexto la UNESCO (2004), considera que "la educación inclusiva es asegurar el derecho a la educación de todos los alumnos, cualesquiera sean sus características o dificultades individuales, a fin de construir una sociedad más justa" (p. 21), siendo clave para alcanzar una verdadera Educación para todos, al considerarse un derecho fundamental en la vida del ser humano.

Es preciso señalar que la educación para todos o la educación inclusiva no se trata de esperar que todos seamos iguales, exigiendo habilidades que otros poseen es imprescindible elaborar un modelo educativo, que reconozca que somos muy diferentes en la forma en que trabajamos, procesamos o transmitimos información, por lo que se debe ser flexibles y diversos. Los niños deben de aprender a respetar y valorar la diversidad, lo cual va a permitir mayores oportunidades de aprendizajes dentro de las aulas, ya que el fin de la educación inclusiva, según Calderón (2014), es el bienestar y el aprendizaje tanto para un niño incluido como regular, por ello es imprescindible el rol del maestro como guía y mediador de los niños dentro del aula (p. 47). Los docentes tienen diferentes roles, es por ello que Navarro-Aburto, B. A., Arriagada Puschel, I. A., Osse-Bustingorry, S., \& Burgos-Videla, C. G., (2016), menciona los siguientes puntos:

1. Educadoras y educadores comprometidos con la formación de sus estudiantes.

2. Educadora y educadores comprometidos con sus responsabilidades docentes.

La labor del docente juega un papel fundamental en la educación inclusiva, por tanto, es necesario reconocer no solo las diferencias de los estudiantes, sino también las habilidades relacionadas con los docentes y su preparación profesional, pues tienen la capacidad de brindar diversos métodos, 
estrategias y medios para los estudiantes en el marco de una buena enseñanza. Al respecto, Meza (2012) define la tarea del docente muy compleja al vincular el currículo con la educación inclusiva por que se requiere combinar la teoría con la práctica para lograr los resultados positivos en todas las actividades de aprendizaje.

\section{Metodología}

La metodología en esta investigación es de corte descriptivo con enfoque cuantitativo, además de la utilización de métodos y técnicas que contribuyeron a la obtención y el análisis de los resultados. Está apoyada con el método bibliográfico y su técnica de recopilación documental mismos que contribuyeron de manera significativa en la parte teórica de la investigación, el método estadístico necesario para graficar los resultados obtenidos y el método deductivo que permitió el análisis de los mismos. La encuesta se la realizó a un total de 20 docentes entre las dos instituciones del cantón Santa Ana - parroquia Santa Ana, Unidad Educativa Fiscal "Ángel Arteaga Cañarte" y Unidad Educativa "9 de Octubre Siglo XXI", basada en la Escala de Likert que contenía 7 preguntas, así mismo la entrevista a 3 profesionales del DECE, misma que contenía 5 preguntas las cuales permitieron responder con el objetivo de esta investigación de analizar las adaptaciones curriculares y su aplicabilidad en la educación inclusiva.

\section{Resultados}

La investigación se la realizó en dos instituciones del cantón Santa Ana ubicadas en la parroquia urbana del mismo nombre, la Unidad Educativa "9 de Octubre Siglo XXI" (10 niños con discapacidad y 12 con necesidades educativas no asociadas a la discapacidad) y la Unidad Educativa Fiscal "Ángel Arteaga Cañarte" (20 estudiantes con discapacidad y 8 con necesidades educativas no asociadas a la discapacidad) aplicando una entrevista y encuesta basada en la Escala de Likert teniendo una valoración de 1: Malo, 2: Bueno, 3: Ni bueno- ni Malo, 4: Muy Bueno y 5: Excelente.

A continuación, se detallan los resultados con la respectiva valoración, frecuencia y porcentajes obtenidos de la encuesta que contenía 7 preguntas aplicadas a 10 docentes de cada institución de manera online, sumando un total de 20 encuestados.

\section{Tabla 1}

Resultados de la encuesta realizada a los docentes de la Unidad Educativa "9 de Octubre Siglo XXI"

Unidad Educativa "9 de Octubre Siglo XXI"

\section{\begin{tabular}{llllll}
\cline { 3 - 5 } Preguntas & VAL. 1 & VAL. 2 & VAL. 3 & VAL. 4 & VAL.5
\end{tabular}}

\begin{tabular}{lccccccccccc} 
& $\mathbf{F}$ & $\%$ & $\mathbf{F}$ & $\%$ & $\mathbf{F}$ & $\%$ & $\mathbf{F}$ & $\%$ & $\mathbf{F}$ & $\%$ & $\%$ \\
\hline $\begin{array}{c}\text { 1. ¿Cómo considera su preparación para la } \\
\text { enseñanza a estudiantes con discapacidad? }\end{array}$ & 1 & $10 \%$ & 3 & $30 \%$ & 1 & 10 & 2 & 20 & 3 & 30 & $100 \%$ \\
\hline
\end{tabular}




\begin{tabular}{|c|c|c|c|c|c|c|c|c|}
\hline 2. ¿Cómo es la coordinación entre el DECE y & $0 \%$ & $10 \%$ & 3 & 30 & 220 & 4 & 40 & $100 \%$ \\
\hline los docentes en referencia a las acciones & & & & $\%$ & $\%$ & & $\%$ & \\
\hline
\end{tabular}
para atender a los estudiantes con N.E.E.?
3. ¿Cómo es la comunicación entre el $\begin{array}{lllllllllll}1 & 10 \% & 1 & 10 \% & 0 & 0 \% & 4 & 40 & 4 & 40 & 100 \%\end{array}$ estudiante con discapacidad y el docente?

$\begin{array}{llllllllllll}\text { 4. ¿Considera que el tiempo brindado al } & 1 & 10 \% & 2 & 20 \% & 3 & 30 & 3 & 30 & 1 & 10 & 100 \% \\ \text { estudiante con N.E.E es suficiente para } & & & & & \% & & \% & & \%\end{array}$
atender los requerimientos del mismo?

\begin{tabular}{|c|c|c|c|c|c|c|c|c|c|c|c|}
\hline $\begin{array}{l}\text { 5. ¿Cómo es el desempeño del estudiante } \\
\text { con discapacidad al aplicar una metodología } \\
\text { adecuada? }\end{array}$ & 0 & $0 \%$ & & $0 \%$ & 5 & $\begin{array}{l}50 \\
\%\end{array}$ & 3 & $\begin{array}{l}30 \\
\%\end{array}$ & 2 & $\begin{array}{l}20 \\
\%\end{array}$ & $100 \%$ \\
\hline $\begin{array}{l}\text { 6. ¿Cree usted que la accesibilidad en la } \\
\text { institución es la apropiada para los } \\
\text { estudiantes con discapacidad? }\end{array}$ & 3 & $30 \%$ & 0 & $0 \%$ & 4 & $\begin{array}{l}40 \\
\%\end{array}$ & 0 & $0 \%$ & 3 & $\begin{array}{l}30 \\
\%\end{array}$ & $100 \%$ \\
\hline $\begin{array}{l}\text { 7. ¿Considera usted que el mobiliario de la } \\
\text { institución para los estudiantes con } \\
\text { discapacidad es el adecuado? }\end{array}$ & 1 & $10 \%$ & 0 & $0 \%$ & 6 & $\begin{array}{l}60 \\
\%\end{array}$ & 2 & $\begin{array}{l}20 \\
\%\end{array}$ & 1 & $\%$ & $100 \%$ \\
\hline
\end{tabular}

Tabla 2

Resultados de la encuesta realizada a los docentes de la "Unidad Educativa Ángel Arteaga Cañarte".

Unidad Educativa Fiscal "Ángel Arteaga Cañarte"

Preguntas

$\begin{array}{lllll}\text { VAL. } 1 & \text { VAL. } 2 & \text { VAL. } 3 & \text { VAL. } 4 & \text { VAL. } 5\end{array}$

\begin{tabular}{cccccccccccc} 
& $\mathbf{F}$ & $\%$ & $\mathbf{F}$ & $\%$ & $\mathbf{F}$ & $\%$ & $\mathbf{F}$ & $\%$ & $\mathbf{F}$ & $\%$ & $\%$ \\
\hline 1. ¿Cómo considera su preparación para la & 0 & $0 \%$ & 2 & $20 \%$ & 4 & $40 \%$ & 3 & $30 \%$ & 1 & $10 \%$ & 100 \\
enseñanza a estudiantes con discapacidad? & & & & & & & & & & $\%$ \\
\hline
\end{tabular}

2. ¿Cómo es la coordinación entre el DECE y $\quad \begin{array}{lllllllllll}10 \% & 1 & 10 \% & 1 & 10 \% & 5 & 50 \% & 2 & 20 \% & 100\end{array}$ los docentes en referencia a las acciones

$\%$ para atender a los estudiantes con N.E.E.?

$\begin{array}{llllllllllll}\text { 3. ¿Cómo es la comunicación entre el } & 0 & 0 \% & 1 & 10 \% & 0 & 0 \% & 6 & 60 \% & 3 & 30 \% & 100\end{array}$ estudiante con discapacidad y el docente?
4. ¿Considera que el tiempo brindado al estudiante con N.E.E es suficiente para

$\begin{array}{llllllllllll}1 & 10 \% & 2 & 20 \% & 3 & 30 \% & 4 & 40 \% & 0 & 0 \% & 100\end{array}$
atender los requerimientos del mismo?

$\begin{array}{llllllllllll}\begin{array}{c}\text { 5. ¿Cómo es el desempeño del estudiante } \\ \text { con discapacidad al aplicar una metodología }\end{array} & 0 & 0 \% & 2 & 20 \% & 0 & 0 \% & 5 & 50 \% & 3 & 30 \% & 100 \\ \end{array}$


adecuada?

\begin{tabular}{|c|c|c|c|c|c|c|c|c|c|c|c|}
\hline $\begin{array}{l}\text { 6. ¿Cree usted que la accesibilidad en la } \\
\text { institución es la apropiada para los } \\
\text { estudiantes con discapacidad? }\end{array}$ & 4 & $40 \%$ & 1 & $10 \%$ & 2 & $20 \%$ & 2 & $20 \%$ & 1 & $10 \%$ & $\begin{array}{l}100 \\
\%\end{array}$ \\
\hline $\begin{array}{l}\text { 7. ¿Considera usted que el mobiliario de la } \\
\text { institución para los estudiantes con } \\
\text { discapacidad es el adecuado? }\end{array}$ & 3 & $30 \%$ & 3 & $30 \%$ & 3 & $30 \%$ & 0 & $0 \%$ & 1 & $10 \%$ & $\begin{array}{l}100 \\
\%\end{array}$ \\
\hline
\end{tabular}

Los resultados obtenidos de la encuesta realizada a los docentes en las dos instituciones indican porcentajes positivos ante la primera interrogante sobre como consideran su preparación para atender las N.E.E, del $100 \%$ de los encuestados en la primera institución respondieron en un $30 \%$ bueno y $30 \%$ excelente y en el segundo centro educativo un $40 \%$ ni bueno-ni malo y un $30 \%$ muy bueno, así mismo en referencia a la segunda pregunta sobre la coordinación y el apoyo del DECE manifestaron que es óptimo pues consideran un $40 \%$ de excelente en la primera institución y un $50 \%$ muy buena en la segunda Unidad Educativa encuestada y es que para el proceso inclusivo reciben asesoría y capacitaciones que les permite cumplir con su rol de educadores comprometidos a brindar una educación inclusiva y de calidad, la tercera pregunta se refiere a la comunicación y en esta ocasión manifestaron en un 40 \% muy bueno y el mismo porcentaje obtuvo la valoración de excelente esto en el primer centro educativo, sin embargo la segunda institución considera $60 \%$ muy bueno y un $30 \%$ excelente.

La cuarta pregunta sobre el tiempo que se le brinda al estudiante con discapacidad para atender sus requerimientos se obtuvieron resultados entre un 30\% con la valoración de ni bueno-ni malo un 30\% muy bueno, mientras que la otra institución refleja porcentajes altos de entre el $30 \%$ y $40 \%$ con la misma valoración. Consideran que es básico utilizar una metodología adecuada para los estudiantes con necesidades educativas especiales, ya que no todos aprenden al mismo ritmo que los niños regulares, por ello se consideró importante preguntar como es el desempeño del estudiante con discapacidad al aplicar una metodología acorde a sus necesidades y capacidades de aprendizaje, los docentes de ambas instituciones ante esta interrogante dieron una valoración positiva en un $50 \%$ ni bueno ni malo, $30 \%$ muy buena y $20 \%$ excelente mientras que en la otra institución refleja un $20 \%$ buena, 50\% muy buena y un $30 \%$ excelente.

La sexta pregunta realizada a los docentes de la Unidad Educativa "9 de Octubre Siglo XXI" que hace referencia si la institución cuenta con la apropiada accesibilidad para los estudiantes con discapacidad ya que la infraestructura y el espacio físico deben de ser flexibles, abiertos y fáciles de transitar, los resultados evidenciaron criterios diferentes, en un $30 \%$ mala, $40 \%$ ni bueno- ni malo y un 
$30 \%$ excelente, así mismo en la Unidad Educativa "Ángel Arteaga Cañarte" el 40\% dio una valoración de malo, $10 \%$ buena, $20 \%$ ni bueno-ni malo, $20 \%$ muy bueno, $10 \%$ excelente. También dentro de las adaptaciones curriculares se deben de tomar en cuenta las condiciones del aula en cuanto a la iluminación, mobiliario y espacio son esencial para el estudiante con discapacidad, por ello se preguntó si consideran que el mobiliario es el adecuado para los estudiantes con discapacidad y los encuestados expresaron en referencia a la primera institución un $10 \%$ mala, $60 \%$ ni bueno-ni malo un $20 \%$ muy buena y un $10 \%$ excelente, a esta interrogante la otra institución evidenció una valoración con criterios divididos en un $30 \%$ malo, $30 \%$ bueno, $30 \%$ ni bueno-ni malo y un $10 \%$ excelente. Todos estos elementos son imprescindibles para facilitar el proceso de aplicación de adaptación curricular en la educación inclusiva.

La entrevista se la realizó a 3 profesionales del DECE, en la Unidad Educativa "9 de Octubre Siglo XXI" a la Psicóloga Educativa Rocío Vinces y en la Unidad Educativa Fiscal "Ángel Arteaga Cañarte", Psicóloga Educativa y Orientadora Familiar Annabelle García quien trabaja en la sección diurna y el Psicólogo Clínico Darwin Cobeña que se encarga de la sección vespertina.

\section{Figura 1}

Resultados de las entrevistas realizadas a los profesionales del DECE

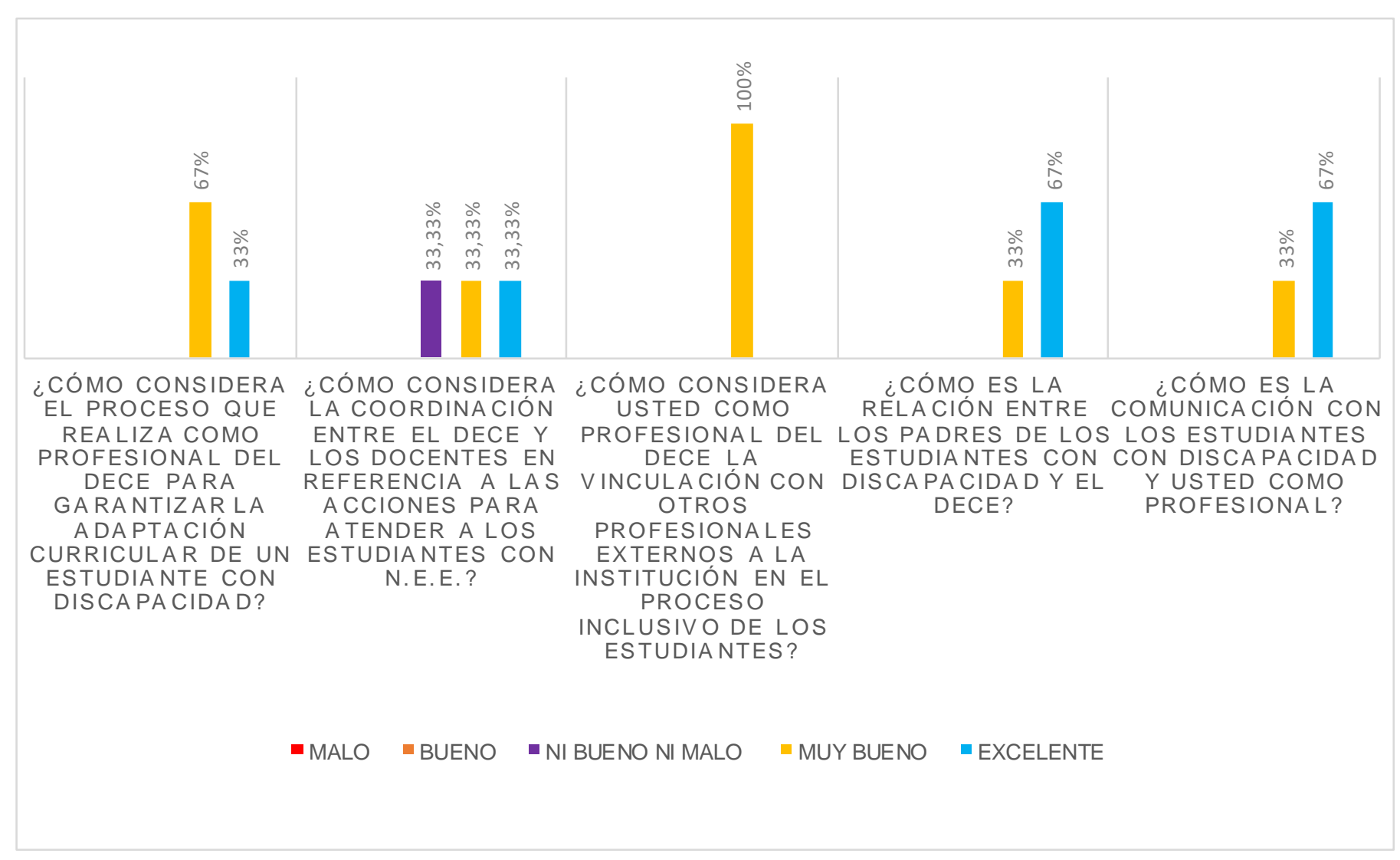


Los resultados en la primera pregunta arrojaron un $67 \%$ de muy bueno y un $33 \%$ de excelente el proceso que realizan para garantizar la aplicación de adaptaciones curriculares al estudiante con NEE siguiendo lo establecido por el Ministerio de Educación del Ecuador aplicando diferentes instrumentos y en ocasiones derivando el caso si este lo amerita. La coordinación entre el DECE y los docentes es necesaria e imprescindible al ser el primero que identifica los problemas de aprendizaje, los profesionales en esta ocasión respondieron de manera diferente al considerar en un 33.33\% ni bueno-ni malo, muy bueno y excelente la coordinación con los maestros, además se realizan capacitaciones y reuniones semanales para dar seguimiento a los estudiantes. El 100\% de los encuestados consideran muy bueno la vinculación con profesionales externos, teniendo el apoyo de diferentes organismos estatales como la UDAI, Junta Cantonal, MSP, entre otras instituciones que son de gran ayuda en el proceso inclusivo dentro de la educación.

En la pregunta sobre la relación que existe entre los padres de familia y el DECE, los entrevistados consideran en un $67 \%$ excelente y un $33 \%$ muy bueno, el mismo porcentaje se evidencia en la quinta pregunta sobre la comunicación con los estudiantes con discapacidad y es que debido a la pandemia mundial por Covid-19 los profesionales brindan de manera virtual contención emocional y así mismo con las respectivas medidas de seguridad realizan visitas domiciliarias en los casos de estudiantes con o sin discapacidad que presentan algún inconveniente para continuar con su educación. Los profesionales no realizan valoración psicológica si no una evaluación diagnóstico de los resultados académicos del alumno. Concuerdan además que todos los estudiantes tienen necesidades especiales pero la cantidad de alumnos que existen en la institución en muchas ocasiones limita su trabajo, por ello pone más énfasis en los estudiantes con discapacidad o los que presenten problemas de aprendizaje.

\section{Discusión}

En el Ecuador se han realizado innumerables esfuerzos creando diversas normativas para lograr una educación inclusiva, sin embargo aún falta mucho por hacer. Esto es un trabajo en conjunto y de corresponsabilidad de toda la comunidad educativa para erradicar la discriminación y exclusión de aquellos estudiantes que poseen necesidades educativas especiales, tengan o no discapacidad, para que puedan ser incluidos en el ambiente educativo y brindar una educación de calidad.

En el Acuerdo Ministerial 0295-13, del Ministerio de Educación, (2013) establece en el artículo 11 que: "La educación inclusiva involucra cambios y modificaciones en contenidos, enfoques, estructura y estrategias con una visión común y la convicción que educar con calidad a todos los niños, niñas y adolescentes del rango de edad apropiado...", para cumplir con este acuerdo es necesario aplicar adaptaciones curriculares a aquellos estudiantes que presenten necesidades educativas, relacionada 0 no a una discapacidad para romper las barreras de aprendizaje en las aulas. Las entrevistas y encuestas 
de manera general evidenciaron que el DECE y las instituciones cumplen con todo el proceso de adaptación curricular hacia los estudiantes con problemas de aprendizaje o alguna discapacidad, contando además con el apoyo de organismos estatales y de los docentes que coordinadamente actúan para garantizar el derecho a una educación inclusiva.

Para potenciar la transformación hacia la educación inclusiva de calidad, Lledó Carreres \& Arnaiz Sánchez (2010), señala que el profesorado es el principal protagonista de las prácticas inclusivas y va a influir en gran medida su formación, actitudes y creencias para un verdadero cambio e innovación en la educación, es importante recalcar que estos deben de tener una adecuada capacitación para tratar casos de estudiantes con problemas de aprendizaje y discapacidad, la comunicación tanto con los padres de familias y los estudiantes debe ser asertiva para generar confianza entre ellos, los docentes encuestados consideran que su preparación es buena para atender estos casos y brindar una enseñanza adecuada, además reciben constantes capacitaciones del DECE y UDAI.

La aplicación de una metodología inclusiva tiene como finalidad la inclusión de todo el alumnado rompiendo los estereotipos y perjuicios en la educación y según Yépez Moreno (2017), la enseñanza requiere de una aplicación seria y contextualizada que reconozca, valore y atienda de manera integral la diversidad, potenciando el desarrollo integral todos los estudiantes. Aplicar una metodología acorde a las condiciones de cada estudiante con NEE es necesaria porque aprenderá de acuerdo a sus capacidades, sin presionarlo a realizar trabajos como un niño regular, los docentes en la encuesta realizada manifestaron que es muy bueno el rendimiento de los estudiantes lo que demuestra que existe un adecuado manejo de la metodología y enseñanza de parte de los docentes. Algo muy importante dentro del proceso de adaptación curricular además de la metodología es que la institución cuente con la accesibilidad y el mobiliario adecuado para las personas que tienen discapacidad, mismos que según consideran algunos docentes este requisito no se cumple en su totalidad en los establecimientos.

\section{Conclusión}

La educación inclusiva es un derecho de todos sin importar sus características, la inclusión es un medio para lograr la igualdad social y una sociedad con igualdad de oportunidades. Las adaptaciones curriculares son muy importantes dentro del proceso de inclusión en las instituciones educativas, logrando identificar y detectar a tiempo aquellos problemas de aprendizaje que presente el estudiante y de esta manera poder realizar las modificaciones para que la enseñanza sea adaptada de acuerdo a las condiciones y diferencias del alumno.

Las instituciones educativas mencionadas en la investigación de manera general cumplen con todo el procedimiento que establece el Ministerio de Educación en la aplicación de adaptaciones curriculares a cada estudiante con NEE, contando con el apoyo de organismos estatales que les permite cumplir con todo el proceso inclusivo, sin embargo muchos docentes según su criterio consideran que no 
existe la adecuada infraestructura de accesibilidad, espacios para la movilidad y mobiliario para los estudiantes con discapacidad, en las instituciones referenciadas para la investigación.

El trabajo en conjunto con la comunidad educativa es esencial para generar un cambio dentro de la educación generando un buen clima institucional, también es importante que los docentes se encuentren capacitados en la aplicación de técnicas y materiales pedagógicos, la utilización de lenguaje inclusivo como lengua de señas y Braille para garantizar el derecho a la educación de los estudiantes con discapacidad.

\section{Referencias Bibliográficas}

Alejandro Conteno, K., Erraéz Alvardo, J., Vargas Gaona, M., \& Espinoza Freire, E. (2018). Consideraciones sobre la educación inclusiva. Revista Metropolitana de Ciencias Aplicadas, 1(3), 18-24. Obtenido de https://remca.umet.edu.ec/index.php/REMCA/article/view/48/154

Asamblea Constituyente. (2008). Constitución de la Republica del Ecuador. Ciudad Alfaro, Montecristi.

Asamblea Nacional Del Ecuador. (2011). Ley Orgánica De Educación Intercultural. Quito: Asamblea Nacional.

Barrio de la Puente, J. (2009). Hacia una Educación Inclusiva para todos. Revista Complutense de Educación, 20(1), 13-21. Obtenido de https://revistas.ucm.es/index.php/RCED/article/view/RCED0909120013A/15360

Calderón, M. (2014). La educación inclusiva es nuestra tarea. RedCDPD, XXI(40), 43-58. Obtenido de http://revistas.pucp.edu.pe/index.php/educacion/article/view/2505/2450

Código de la Niñez y Adolescencia. (2017).

Crisol, E., Martínez, J., \& El Homrani, M. (Noviembre de 2015). El aula inclusiva. Condiciones didáctica y organizativas. Revista nacional e internacional de educación inclusiva, 8(3), 254-270. Obtenido de file://C:/Users/MELISSA/Downloads/Dialnet-

ElAulalnclusivaCondicionesDidacticaYOrganizativas-5446541.pdf

Crosso, C. (2014). El derecho a la educación de personas con discapacidad. Impulsando el concepto de educación inclusiva. RedCDPD, $17 . \quad$ Obtenido de http://repositoriocdpd.net:8080/bitstream/handle/123456789/413/Art_CrossoC_DerechoEducacio nPersonas_2010.pdf?sequence $=1$

Galve, J. L., \& Trallero, M. (2002). Adaptaciones curriculares. Guía para profesores tutores de educación primaria y educación especial. España: CEPE.

León Guerrero, M. (2011). La situación de la formación en educación inclusiva en los nuevos títulos de grado de maestro en España. Revista Interuniversitaria de Formación del Profesorado, 25(1), 145-163. Obtenido de https://www.redalyc.org/pdf/274/27419147009.pdf 
Lledó Carreres, A., \& Arnaiz Sánchez , P. (2010). Evaluación de las Prácticas Educativas del Profesorado de los Centros Escolares: Indicadores de Mejora desde la Educación Inclusiva. REICE. Revista Iberoamericana sobre Calidad, Eficacia y Cambio en Educación, 8(5), 96-109. Obtenido de https://revistas.uam.es/reice/article/view/4729/5163

Llerena Companioni, O., \& Salinas Martínez, C. (2018). Inclusión educativa y adaptaciones curriculares en el proceso de enseñanza aprendizaje. Machala: UTMACH. Obtenido de https://www.researchgate.net/profile/Odalia_Llerena2/publication/326096423_Inclusion_educativ a_y_adaptaciones_curriculares_en_el_proceso_de_ensenanza_aprendizaje/links/5b4374dea6fd ccbcf90f80aa/Inclusion-educativa-y-adaptaciones-curriculares-en-el-proceso-

Meza, J. (2012). Diseño y Desarrollo Curricular. Estado de Mexico: RED TERCER MILENIO S.C. Obtenido http://www.aliat.org.mx/BibliotecasDigitales/derecho_y_ciencias_sociales/Diseno_y_desarrollo_c urricular.pdf

Ministerio de Educación. (15 de Agosto de 2013). Acuerdo Ministerial 0295-13. Quito, Ecuador. Obtenido de https://educacion.gob.ec/wp-content/uploads/downloads/2013/08/ACUERDO_295-13.pdf

Ministerio de Educación. (14 de Octubre de 2014). Unidad Distrital de Apoyo a la Inclusión UDAl. Obtenido de https://educacion.gob.ec/unidad-de-apoyo-a-la-inclusion-udai/

Ministerio de Educación del Ecuador. (2016). Guia de trabajo: Adaptaciones curriculares para la educacion especial e inclusiva. 81. Obtenido de https://educacion.gob.ec/wpcontent/uploads/downloads/2019/05/Guia-de-adaptaciones-curriculares-para-educacioninclusiva.pdf

Ministerio de Educación del Ecuador. (2020). Guía de apoyo pedagógico para la comunidad educativa durante el periodo de suspención de las actividades escolares presenciales por la emergencia sanitaria COVID-19. Quito. Obtenido de https://educacion.gob.ec/wpcontent/uploads/downloads/2020/09/Guia-de-apoyo-pedagogico-para-la-Comunidad-

Educativa.pdf

Navarro-Aburto, B. A., Arriagada Puschel, I. A., Osse-Bustingorry, S., \& Burgos-Videla, C. G. (2016). Adaptaciones curriculares: Convergencias y divergencias de su implementación en el profesorado chileno. Revista Electrónica Educare, 20(1), 18. Obtenido de https://doi.org/10.15359/ree.20-1.15

Paniagua, C. (2005). Las Adaptaciones curriculares: concepto y alcances en el marco de la integración escolar. Revista argentina de psicopedagogía(59). Obtenido de file://C:/Users/MELISSA/Downloads/Dialnet-LasAdaptacionesCurriculares-2057954\%20(3).pdf

Payà, A. (2010). Políticas de educación inclusiva en América Latina Propuestas, realidades y retos de futuro. Revista Educación Inclusiva, 3(2), 125-142. Obtenido de https://revistaeducacioninclusiva.es/index.php/REl/article/view/209/203\# 
Plancarte, P. A. (2017). Inclusión educativa y cultura inclusiva. Revista Nacional e Internacional de Educación Inclusiva, 10(2), 213-226. Obtenido de file://C:/Users/MELISSA/Downloads/DialnetInclusionEducativaYCulturalnclusiva-6545223\%20(1).pdf

Posada Silva, W.Y.; Marín Giraldo, Y. y Gómez Chica, S. (2015). La diversidad funcional y las adaptaciones curriculares. Revista de Investigaciones. UCM, 15(26), 192-202. doi: http://dx.doi.org/10.22383/ri.v15i2.55

Reglamento General a la Ley Orgánica de Educación Intercultural. (2012). Quito, Ecuador.

Rodríguez Hernández, H. J. (Junio de 2016). La Educación Inclusiva en la Agenda Educativa Mundial 2015-2030. Revista nacional e internacional de educación inclusiva, 9(2), 1-17. Obtenido de https://revistaeducacioninclusiva.es/index.php/REl/article/viewFile/47/43

UNESCO. (2004). Temario abierto sobre educación inclusiva: materiales de apoyo para responsables de políticas educativas. Santiago: Impreso por Archivos Industriales y Promocionales Ltda. Obtenido de https://sid.usal.es/idocs/F8/FDO23200/temario_abierto_educacion_inclusiva.pdf

Yépez Moreno, A. G. (2017). Metodologías Inclusivas en el Contexto Socio-Educativo. Retos de la Ciencia, 1(2), 131-139. 